\title{
One-Pot Electrochemical Synthesis of Hierarchical Porous Niobium
}

\author{
Gihwan Joe and Heon-Cheol Shin* \\ School of Materials Science and Engineering, Pusan National University, Busandaehak-ro 63 beon-gil, Geumjeong-gu, \\ Busan 46241, South Korea
}

\begin{abstract}
In this study, we report niobium $(\mathrm{Nb})$ with hierarchical porous structure produced by a one-pot, HF-free electrochemical etching process. It is proved experimentally that a well-defined hierarchical porous structure is produced from the combination of a limited repetition of pulse etching and high concentration of aggressive anion (i.e., $\mathrm{SO}_{4}{ }^{2-}$ ), which results in hierarchical pores with high order over 3 . A formula is derived for the surface area of porous $\mathrm{Nb}$ as a function of the hierarchical order of pores while the experimental surface area is estimated on the basis of the electrochemical gas evolution rate on porous $\mathrm{Nb}$. From the comparison of the theoretical and experimental surface areas, an in-depth understanding was gained about porous structure produced in this work in terms of the actual pore shape and hierarchical pore order.
\end{abstract}

Keywords : Electrochemical Etching, HF-free, Niobium, Hierarchical Pore Structure, Fractal

Received : 7 December 2020, Accepted : 22 December 2020

\section{Introduction}

Because of its variable oxidation states, $\mathrm{Nb}$, a transition metal, can react with various elements such as oxygen, carbon, and nitrogen to form a wide range of functional compounds. One such compound is $\mathrm{Nb}_{2} \mathrm{O}_{5}$, which has received significant attention as an electrode material for sensors [1-4], dye-sensitized solar cells [5], photodetectors [6], and capacitors $[7,8]$, because of its large band-gap (3.2 4 eV) [9] and high dielectric constant ( 41) [10]. Moreover, it can be applied as an electrode material in lithium secondary batteries because of its excellent energy storage per unit volume as well as its suitable charge and discharge potentials $[11,12]$.

Porous materials are known to have numerous benefits, particularly when used as electrodes in functional electrochemical devices. Because of their large surface areas [13-16], these electrodes often show improved capacitance, extraordinary sensitivity, and increased power when used in capacitors [17,18], sensors [19], and secondary batteries, respectively

*E-mail address: hcshin@pusan.ac.kr

DOI: https://doi.org/10.33961/jecst.2020.01725

This is an open-access article distributed under the terms of the Creative Commons Attribution Non-Commercial License (http://creativecommons.org/licenses/by-nc/4.0) which permits unrestricted non-commercial use, distribution, and reproduction in any medium, provided the original work is properly cited.
[20,21]. In addition, the low fill factor, i.e., the large free volume of porous materials, facilitates the rapid movement of ions/atoms through the material, possibly suppressing the concentration overpotential $[13,21]$. Thus, the efficiency of the devices is significantly improved, particularly when mass transport is the rate-determining process [14]. A large free volume can also enhance the durability of the device by serving as a buffer against the possible expansion or contraction of the material during operation [14,22,23].

Various wet-chemical methods such as hydrothermal synthesis, sol-gel processing, anodic oxidation, and chemical/electrochemical etching have been used for the preparation of porous materials. Among these, the electrochemical methods are cost-effective, as they involve simple device configuration and easy control of the process variables (i.e., current and voltage). In addition, they aid in obtaining highly reproducible results. Thus, the electrochemical fabrication of a wide range of porous materials has been extensively studied. Porous $\mathrm{Nb}$ structures have been obtained via the anodic oxidation of $\mathrm{Nb}$ [24-26]. However, this process either requires the use of a toxic chemical, HF, in an aqueous solution [27] or high-temperature conditions $\left(>160^{\circ} \mathrm{C}\right)$ in a non-aqueous solution [28]. Additionally, the product is limited 
to a porous $\mathrm{Nb}$ oxide. Electrochemical etching is another well-known and cost-effective method that can be used to prepare a porous Nb structure [7,29]. However, HF is also commonly used in this process, although it has been reported that an appropriate combination of HF with different types of organic solvents can allow for good control over the formation of the porous Nb surface [29].

In this study, a porous $\mathrm{Nb}$ structure was prepared via a one-pot, HF-free, electrochemical etching process. In particular, a novel hierarchical porous $\mathrm{Nb}$ or self-similar fractal porous $\mathrm{Nb}$ structure was successfully obtained using the pulse etching. Initially, the effect of the applied current density on the uniformity and density of the pores was examined. Subsequently, critical factors, namely, the type of etching process (continuous $v s$. pulse) and the etchant concentration, affecting the hierarchical order of the pores were optimized. In addition, the surface area of the porous structure was theoretically evaluated as a function of the hierarchical pore order. The actual pore shape and hierarchical pore order were examined on the basis of this theoretical study and the obtained experimental results.

\section{Experimental}

For the electrochemical etching of $\mathrm{Nb}$, a two-electrode electrochemical cell was constructed using an $\mathrm{Nb}$ foil (Alfa Aesar, $127 \mu \mathrm{m}$, annealed, 99.8\%) and a Pt mesh as the working and counter electrodes, respectively. Methanol (Alfa Aesar, 99\%) with $1 \mathrm{M}$ or $1.5 \mathrm{M} \mathrm{H}_{2} \mathrm{SO}_{4}$ (Reagents Duksan, Korea) was used as an etching solution. The exposed area of an $\mathrm{Nb}$ foil to a solution was $1 \mathrm{~cm}^{2}$. The distance between the two electrodes was kept at $1 \mathrm{~cm}$.

Electrochemical etching of $\mathrm{Nb}$ was conducted in the following three cases:

(1) Application of constant anodic current densities ranging from 30 to $100 \mathrm{~mA} \mathrm{~cm}^{-2}$ for $10 \mathrm{~min}$ in a methanolic solution of $1 \mathrm{M} \mathrm{H}_{2} \mathrm{SO}_{4}$ for analyzing the effect of etching current on the surface morphology of $\mathrm{Nb}$;

(2) A $10 \mathrm{~min}$ application of a $50 \mathrm{~mA} \mathrm{~cm} \mathrm{~cm}^{-2}$ current density, a $1 \mathrm{~min}$ rest time, and an additional $5 \mathrm{~min}$ application of the $50 \mathrm{~mA} \mathrm{~cm}{ }^{-2}$ current density, followed by the repetition of the latter two processes, i.e., 'rest time and additional current application'. 1 to 300 repetitions of pulse etching in a methanolic solution of $1 \mathrm{M} \mathrm{H}_{2} \mathrm{SO}_{4}$ for creating a hierarchical porous structure;

(3) 10 repetitions of pulse etching in a methanolic solution of $1.5 \mathrm{M} \mathrm{H}_{2} \mathrm{SO}_{4}$ for improving a uniformity and hierarchical order of porous structure (periodical on and off of anodic etching current density was kept the same as in the case (2))

The surface morphology and composition of the $\mathrm{Nb}$ samples were analyzed using field-emission scanning electron microscope (FE-SEM, MIRA3, TESCAN, Czech Republic) and energy dispersive X-ray spectrometer (EDS, 51-XMX1004, Oxford instruments, UK). To experimentally quantify their surface area, the electrochemical reduction of $\mathrm{H}^{+}$(i.e., $\mathrm{H}_{2}$ generation) was conducted on their surface. For this purpose, a three-electrode electrochemical cell was constructed, where the $\mathrm{Nb}$ was used as the working electrode, a Pt mesh and a saturated calomel electrode (SCE) were adopted for the counter and reference electrodes, respectively. The reduction of $\mathrm{H}^{+}$ was carried out at the applied potential of $-1.0 \mathrm{~V} v s$. SCE for $100 \mathrm{~s}$ in an aqueous solution of $0.1 \mathrm{M}$ $\mathrm{H}_{2} \mathrm{SO}_{4}$. All of the etching and electrochemical $\mathrm{H}_{2}$ generation experiments were conducted using IVIUMSTAT instrument (Ivium Technologies B.V., The Netherlands).

\section{Results and Discussion}

Fig. 1 shows the morphology of the porous $\mathrm{Nb}$ surfaces etched in a methanolic solution of $1 \mathrm{M} \mathrm{H}_{2} \mathrm{SO}_{4}$ for $10 \mathrm{~min}$ at anodic current densities ranging from 30 to $100 \mathrm{~mA} \mathrm{~cm}^{-2}$. The application of a low current density, $30 \mathrm{~mA} \mathrm{~cm}^{-2}$, led to the formation of a lowdensity porous structure, wherein pores were formed only on selected regions of the surface (Fig. 1a, b). Increasing the current density to $50 \mathrm{~mA} \mathrm{~cm}^{-2}$ created a relatively uniform porous structure with pore diameters of approximately $25 \mu \mathrm{m}$ (Fig. 1c, d); however, a further increase in the current density to $100 \mathrm{~mA} \mathrm{~cm}^{-2}$ resulted in excessive etching with severe overlap among the pores (Fig. 1e, f).

Pore formation during electrochemical etching progresses via pore nucleation and subsequent pore growth. Notably, the aggressive anions in the etching solution preferentially interact with the energetically activated local domains to induce pore nucleation. In many previous studies on electrochemical etching using etchants containing $\mathrm{HF}$ or $\mathrm{F}^{-}$, highly localized 


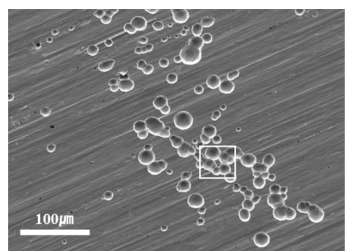

(a)

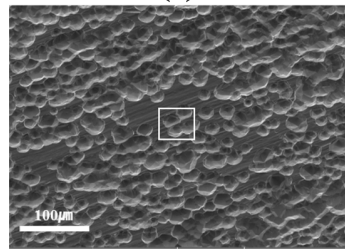

(c)

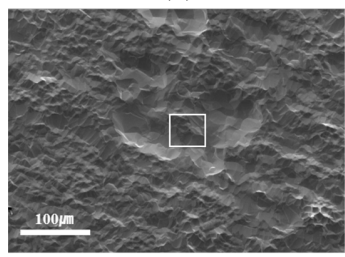

(e)

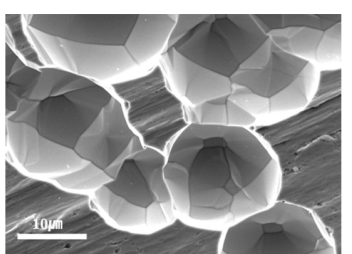

(b)

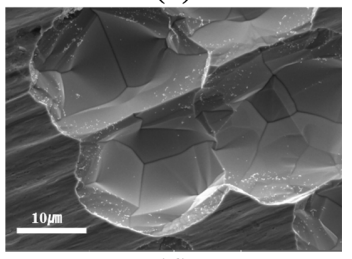

(d)

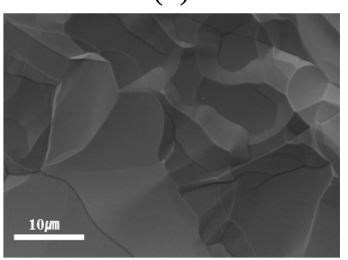

(f)
Fig. 1. The morphology of the porous $\mathrm{Nb}$ surfaces etched in a methanolic solution of $1 \mathrm{M} \mathrm{H}_{2} \mathrm{SO}_{4}$ for $10 \mathrm{~min}$ at different anodic current densities : (a), (b) $30 \mathrm{~mA} \mathrm{~cm}^{-2}$, (c), (d) $50 \mathrm{~mA} \mathrm{~cm}^{-2}$ and (e), (f) $100 \mathrm{~mA} \mathrm{~cm}^{-2}$

dissolution of the metal surface was observed, which resulted in the formation of pores with small diameters and high aspect ratios [30,31]. However, despite their utility in the formation of porous structures with large surface areas, the use of chemicals containing $\mathrm{F}^{-}$ ions should be avoided because they are hazardous to the environment. Therefore, in this study, $\mathrm{H}_{2} \mathrm{SO}_{4}$ was used as the etchant and the $\mathrm{SO}_{4}{ }^{2-}$ ions behaved as the aggressive anions. It was hypothesized that the relatively low aggressiveness of $\mathrm{SO}_{4}{ }^{2-}$ compared to that of $\mathrm{F}^{-}$would result in the formation of pores with larger diameters, as the regions of dissolution on the metal surface would be broader when $\mathrm{SO}_{4}{ }^{2-}$ is used. The experimental results were found to be in good accordance with this hypothesis (Fig. 1). Pore nucleation and growth induced by $\mathrm{SO}_{4}{ }^{2-}$ might occur via a mechanism similar to that of localized corrosion, as follows: (1) localized destruction of the passivation film on the metal surface by $\mathrm{SO}_{4}{ }^{2}$; (2) electrochemical oxidation (dissolution) of the metal and subsequent pore nucleation; (3-1) entry of $\mathrm{SO}_{4}{ }^{2-}$ into the pores due to its electrostatic attraction to the dis- solved metal cations; (3-2) formation of metal sulfate, followed its hydrolysis to produce the metal hydroxide and sulfuric acid, thus increasing the acidity of the pores; (4) pore wall dissolution (pore growth) due to the increased acidity and $\mathrm{SO}_{4}{ }^{2-}$ concentration.

Thus, it is apparent that during pore formation, the pore growth rate is significantly affected by the concentrations of the $\mathrm{SO}_{4}{ }^{2-}$ and $\mathrm{H}^{+}$ions inside the pores, which are influenced by the concentration of the metal cations inside the pores. The duration for which the dissolved metal cations remain in the pores depends on the effective porosity of the pores. If the pores have a relatively closed conformation, then it is challenging for the metal cations to diffuse out, thus promoting the inflow of anions and pore growth. In contrast, open pores facilitate the diffusion of the metal cations, thus suppressing the inflow of anions and preventing pore growth. The pores formed in this study seemed to have an open conformation, indicating that the concentration of the dissolved $\mathrm{Nb}$ ions in the pores was low during electrochemical etching. This hindered the pore growth and suggested that optimizing the etching conditions could result in the formation of remarkably small pores with compact structures.

Current density provides the electrochemical driving force for pore formation, making it a critical factor to consider for the controlled growth of the pore structure. However, from the numerous experiments performed under various conditions, it was observed that the continuous application of a constant current density alone could not result in the formation of an ideal porous structure, i.e., a rough $\mathrm{Nb}$ surface with large specific surface area. Small-diameter, low-density pores were formed at a low current density (30 $\mathrm{mA} \mathrm{cm}{ }^{-2}$ ) while large-diameter, severely overlapped pores were obtained at a high current density (100 $\mathrm{mA} \mathrm{cm}{ }^{-2}$ ), as shown in Fig. 1. Therefore, the optimized current density was chosen to be $50 \mathrm{~mA} \mathrm{~cm}^{-2}$, so that a relatively compact and uniform pore structure was formed. Other factors affecting the specific surface area of the pore structures were subsequently optimized at this current density.

The open conformation of the pores is generally stable and difficult to modify. Thus, the open pores formed by the application of the optimized current density (henceforth referred to as the primary pores) can be considered to be the framework for the forma- 
tion of a hierarchical porous structure, which can significantly increase the specific surface area of the porous material. The open conformation of the pores and pore nucleation results from the interaction of the aggressive ion, $\mathrm{SO}_{4}{ }^{2-}$, with the activated local domains. However, the concentration of $\mathrm{SO}_{4}{ }^{2-}$ inside the pores is substantially low due to the difficulty in the migration of these ions into the open pores. Moreover, even if diffusion did occur, it is unlikely that the shortage of $\mathrm{SO}_{4}{ }^{2-}$ ions would be relieved, as the electrochemical $\mathrm{Nb}$ etching is thought to follow the typical diffusion-controlled mechanism.

Thus, it is assumed that electrochemical etching under a continuous application of current would lead to the formation of primary pores only. Therefore, to obtain a hierarchical porous structure, the high $\mathrm{SO}_{4}{ }^{2-}$ concentration must be maintained throughout the etching process in order to continuously induce pore nucleation, and thus, pore growth on the porous surface. This can be achieved by the pulse etching method, which facilitates the supply of the reactants to the pore surface during the rest time between etching processes. Thus, a high $\mathrm{SO}_{4}{ }^{2-}$ concentration is reestablished, and pore nucleation is successfully induced during the subsequent re-etching process.

Figs. 2a and $\mathrm{b}$ show the surface morphology of $\mathrm{Nb}$ obtained via the pulse etching process, which involved the application of a $50 \mathrm{~mA} \mathrm{~cm}{ }^{-2}$ current density for 10 $\mathrm{min}$, followed by a $1 \mathrm{~min}$ rest time, and then, an additional $5 \mathrm{~min}$ application of the $50 \mathrm{~mA} \mathrm{~cm}^{-2}$ current density in a methanolic solution of $1 \mathrm{M} \mathrm{H}_{2} \mathrm{SO}_{4}$. Interestingly, the formation of small pores (secondary pores) on the walls of the large pores (primary pores) could be observed even after the first pulse cycle, i.e., one repetition of 'the rest time followed by an additional 5 min current application'. This observation strongly supports the hypothesis stated earlier; hierarchical pores can be formed through the pulse etching of $\mathrm{Nb}$ performed at the optimized conditions. Lowdensity secondary pores were formed after the application of the first pulse cycle, possibly due to the rapid consumption of the additional $\mathrm{SO}_{4}{ }^{2-}$ ions that were supplied during the rest time. Thus, the number of pulse cycles was increased to examine the pattern formation of the small pores. Figs. $2 c$ and d show the surface morphology of the porous $\mathrm{Nb}$ obtained from ten pulse cycles. High-density secondary pores with diameters of approximately $<1 \mu \mathrm{m}$ were successfully created with negligible overlap between the second-

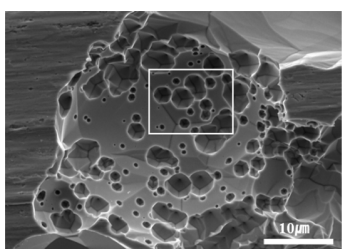

(a)

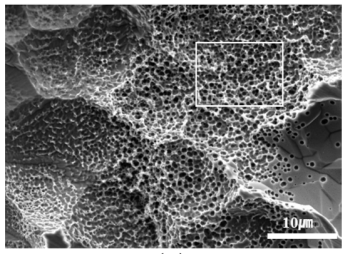

(c)

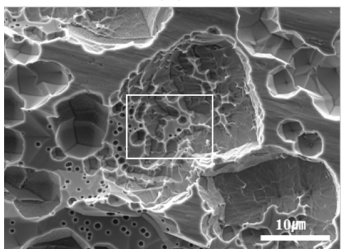

(e)

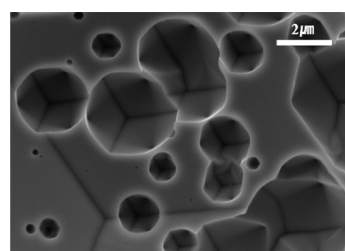

(b)

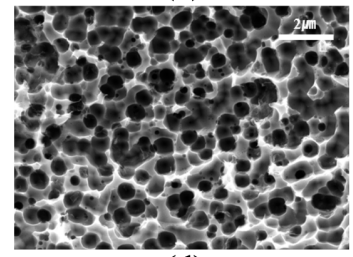

(d)

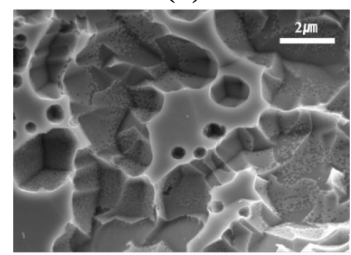

(f)
Fig. 2. The surface morphology of $\mathrm{Nb}$ obtained via the pulse etching process, which involved the application of a 50 $\mathrm{mA} \mathrm{cm}{ }^{-2}$ current density for $10 \mathrm{~min}$, followed by a $1 \mathrm{~min}$ rest time, and an additional $5 \mathrm{~min}$ application of the $50 \mathrm{~mA} \mathrm{~cm}^{-2}$ in a methanolic solution of $1 \mathrm{M} \mathrm{H}_{2} \mathrm{SO}_{4}$. The number of pulse etching (i.e., rest time and additional current application) is 1 at (a), (b), 10 at (c), (d) and 300 at (e), (f).

ary pores. However, after 300 pulse cycles, the uniformity of the pores drastically degraded (Fig. 2e, f), and insignificant amounts of high-order hierarchical pores were created. This implies that increasing the number of pulse cycles when the concentration of $\mathrm{SO}_{4}{ }^{2-}$ is $1 \mathrm{M}$ leads to over-etching of the low-order (i.e., primary and secondary) pores, thus causing the overall framework of the porous structure to collapse. In addition, increasing the number of pulse cycles does not facilitate the nucleation of high-order pores with much smaller sizes.

Therefore, in order to achieve a well-defined, highorder hierarchical porous structure, the nucleation of high-order pores needs to be promoted while the over-etching of low-order pores should be prevented during the etching process. Thus, the initial $\mathrm{SO}_{4}{ }^{2-}$ concentration was increased to allow the pulse etching to proceed in a more aggressive etching environment, and the experiment was performed only till 10 pulse cycles. Figs. 3a, b and c show the surface, 


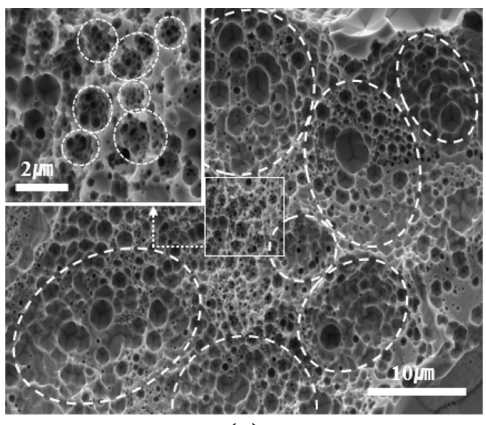

(a)

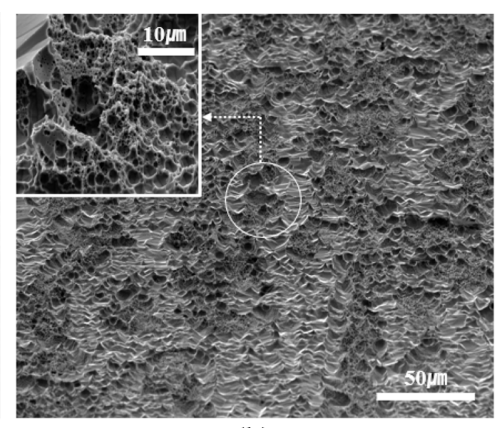

(b)

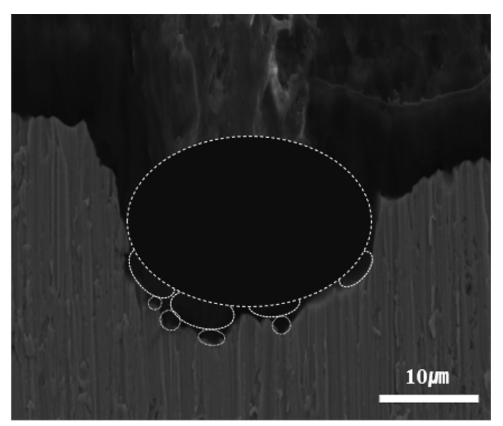

(c)

Fig. 3. (a) Surface, (b) inclined and (c) cross-sectional images of the porous Nb structure obtained from 10 repetitions of pulse etching in a methanolic solution of $1.5 \mathrm{M} \mathrm{H}_{2} \mathrm{SO}_{4}$ (Insets in (a) and (b): magnified images).

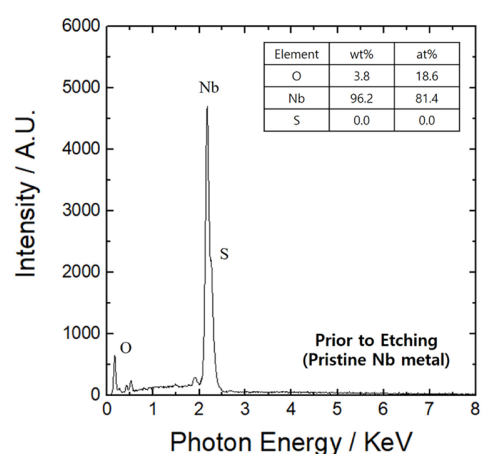

(a)

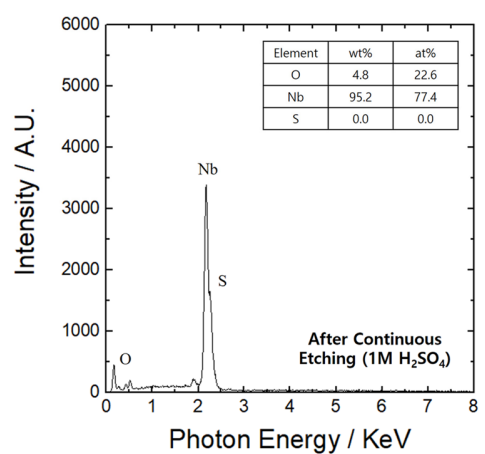

(b)

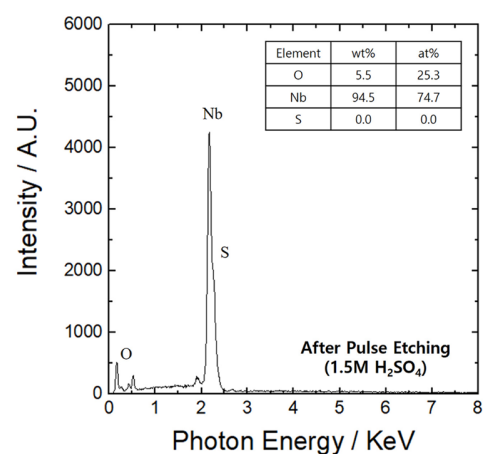

(c)

Fig. 4. Compositional analysis of the $\mathrm{Nb}$ surface by energy-dispersive X-ray spectroscopy. (a) Prior to etching (pristine $\mathrm{Nb}$ metal). (b) After continuous etching at $1 \mathrm{M} \mathrm{H}_{2} \mathrm{SO}_{4}$ solution. (c) After pulse etching at $1.5 \mathrm{M} \mathrm{H}_{2} \mathrm{SO}_{4}$ solution.

inclined, and cross-sectional images of the porous $\mathrm{Nb}$ structure obtained from 10 repetitions of pulse etching in a methanolic solution of $1.5 \mathrm{M} \mathrm{H}_{2} \mathrm{SO}_{4}$. Surprisingly, simply increasing the $\mathrm{SO}_{4}{ }^{2-}$ concentration during pulse etching led to the formation of homogeneous, hierarchical pore structures. The formation of tertiary pores was verified through electron microscopy, although higher order pore structures may have also been created. Although this hierarchical pore structure involves rather limited iteration, it can be viewed as a fractal porous structure with self-similarity.

It is further noted that the surface composition of the porous $\mathrm{Nb}$ samples, obtained from continuous and pulse etching, was almost the same as that of the pristine $\mathrm{Nb}$ metal (i.e., the sample prior to etching), as shown in Fig. 4. This strongly indicates any oxides or sulfates were not formed on the $\mathrm{Nb}$ surface during the etching process. Oxygen detected both on the pristine and etched $\mathrm{Nb}$ samples might originate from the native oxide layer spontaneously formed when they were exposed to the atmosphere.

This study also proposes a theoretical approach for the in-depth analysis of the surface area as a function of the hierarchical order of pores. The overall surface area of a flat porous material can be expressed as follows:

$$
S_{\text {total }, n}=S_{\text {porous }, n}+S_{\text {non-porous }, n}
$$

where $n$ represents the hierarchical order of the pore structure. $S_{\text {total }, n}, S_{\text {porous }, n}$, and $S_{\text {non-porous }, n}$ represent the total surface areas of the porous material, pores, and free material, i.e., the area of the material without pores, respectively. Geometrically, when a 2D surface is densely packed with a hexagonal arrangement of circles, the circles occupy approximately 
$90.7 \%$ of the surface, regardless of their diameter, leaving a free area of $9.3 \%$. This concept was used to simplify the calculations; it was assumed that the pores had circular openings and that they were densely packed and hexagonally arranged. As reflected by the experimental results, the pores were assumed to have an oblate spheroidal shape, wherein the major axis (pore diameter) and minor axis (pore depth) differed in length (Fig. 3c). Another assumption used was that the pores were not formed on the area excluded from the hexagonal arrangement even when the hierarchical pore order was increased.

Under these assumptions, the area of the pores in the $n^{\text {th }}$ hierarchical order can be calculated by the following mathematical equations. After the initial pore formation process, $N_{1}$, the number of pores with $n=1$ (primary pores), can be evaluated as

$$
N_{1}=\frac{0.907 S_{0}}{\pi r_{1}^{2}}
$$

where $S_{0}$ is the initial surface area of the material and $r_{1}$ is the radius of the primary pores. Thus, the surface area of the primary pores with a presumed oblate spheroidal shape, $A_{\text {oblate }, 1}$, is

$$
A_{\text {oblate }, 1}=2 \pi r_{1}^{2}+\frac{\pi c_{1}^{2}}{e_{1}} \cdot \ln \left(\frac{1+e_{1}}{1-e_{1}}\right), e_{1}=\sqrt{1-\frac{c_{1}^{2}}{r_{1}^{2}}}
$$

where $r_{1}$ and $c_{1}$ represent the lengths of the major axis (pore diameter) and minor axis (pore depth) of the primary pores, respectively, while $e_{1}$ indicates the eccentricity [32]. When the second term of Eq. (3) is formatted such that it obtains a form similar to that of the first term, the equation is simplified to

$$
A_{\text {oblate }, 1}=k_{1} \pi r_{1}^{2}
$$

where $k_{1}$ is a numerical value obtained by applying the surface area calculated using $r_{1}, c_{1}$, and $e_{1}$ in Eq. (3) to Eq. (4). Hence, the overall surface area occupied by only the primary pores can be expressed as follows:

$$
S_{\text {porous }, 1}=A_{\text {oblate }, 1} \times N_{1}=0.907 k_{1} S_{0}
$$

It is also important to consider the surface area occupied by the secondary or higher-order pores. When the secondary pores are arranged in a hexagonal manner on the rough surface of the primary pores, they occupy only $90.7 \%$ of the primary pore surface. Thus, the number of secondary pores formed on the primary pores, $N_{2}$, and the area of secondary pores, $A_{\text {oblate, } 2 \text {, can be expressed as }}$

$$
\begin{gathered}
N_{2}=\frac{0.907 S_{\text {porous }, 1}}{\pi r_{2}^{2}} \\
A_{\text {oblate }, 2}=k_{2} \pi r_{2}^{2}
\end{gathered}
$$

Hence, the surface area of the secondary pores can be calculated as

$$
\begin{aligned}
S_{\text {porous }, 2} & =A_{\text {oblate }, 2} \times N_{2} \\
& =0.907 k_{2} S_{\text {porous }, 1}=0.907^{2} k_{1} k_{2} S_{0}
\end{aligned}
$$

Therefore, the general formula for the surface area of $n^{\text {th }}$-order pores can be expressed as follows:

$$
S_{\text {porous }, n}=A_{\text {oblate }, n} \times N_{n}=0.907^{n}\left(\prod_{i=1}^{n} k_{i}\right) S_{0}
$$

An area corresponding to $9.3 \%$ of the total surface area of the $n^{\text {th }}$-order pores will always be excluded from the area of the $n+1^{\text {th }}$-order pores. This is the area of the free material, i.e., $S_{\text {non-porous, },}$, and it can be expressed as

$$
\begin{aligned}
& S_{\text {non-porous }, n}=0.093\left(S_{0}+\sum_{i=2}^{n} S_{\text {porous }, i-1}\right)(\mathrm{n} \geq 2) \\
& \left(S_{\text {non-porous }, 1}=0.093 S_{0}\right)
\end{aligned}
$$

Assuming that the value of $k$ is constant, irrespective of the pore order and the initial surface area is set to unity $\left(S_{0}=1 \mathrm{~cm}^{2}\right)$, the total surface area, $S_{\text {total, } n}$ (unit: $\mathrm{cm}^{2}$ ) can be expressed as

$$
\begin{aligned}
& S_{\text {total }, n}=S_{\text {porous }, n}+S_{\text {non- porous }, n} \\
& =(0.907 k)^{n}+0.093\left\{\begin{array}{c}
n \\
1+\sum_{i=2}^{n}(0.907 k)^{i-1}
\end{array}\right\}(\mathrm{n} \geq 2) \\
& \left(S_{\text {total }, 1}=S_{\text {porous }, 1}+S_{\text {non-porous }, 1}=0.907 k+0.093\right)
\end{aligned}
$$

To experimentally evaluate the surface area of the porous $\mathrm{Nb}$, the electrochemical reduction of $\mathrm{H}^{+}$on the $\mathrm{Nb}$ surface was conducted in $0.1 \mathrm{M} \mathrm{H}_{2} \mathrm{SO}_{4}$ solution for $100 \mathrm{~s}$, at an applied potential of $-1.0 \mathrm{~V} v \mathrm{~s}$. SCE. Fig. 5a shows the comparison between the rate of $\mathrm{H}^{+}$reduction on the flat $\mathrm{Nb}$ surface prior to etching 


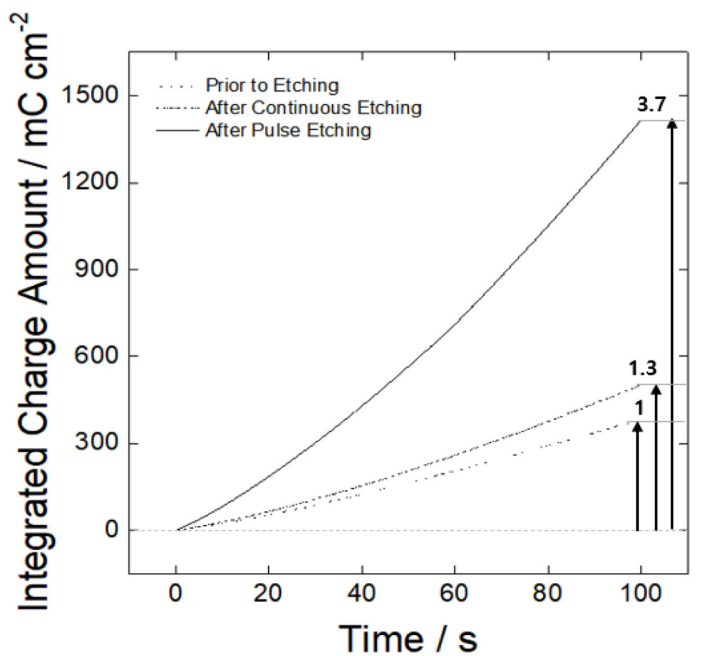

(a)

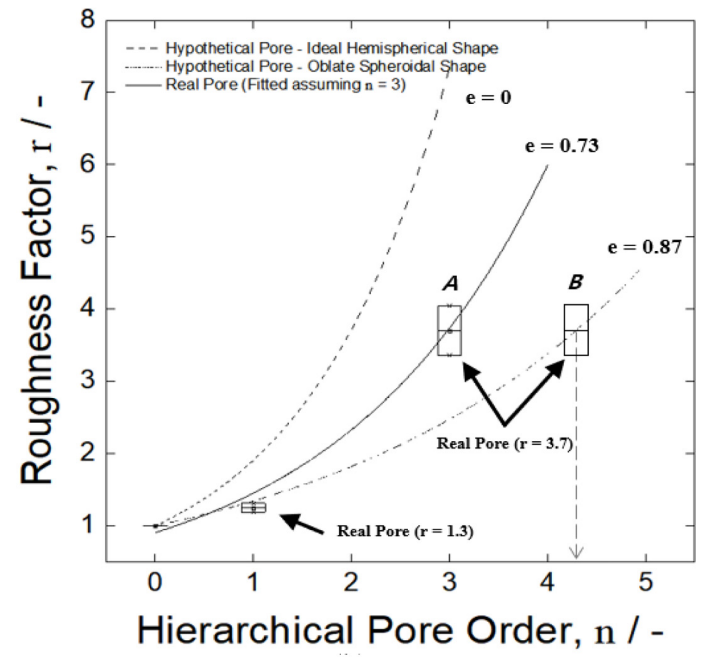

(b)

Fig. 5. (a) The rates of $\mathrm{H}^{+}$reduction on the flat $\mathrm{Nb}$ surface prior to etching and the porous $\mathrm{Nb}$ surfaces obtained from the continuous (Fig. 1(c), (d)) and pulse etching (Fig. 3) processes. (b) The dependence of roughness factor $r$ on the hierarchical pore order $n$ for the hypothetical and experimentally-obtained pores.

and the rates on the porous surfaces obtained from the continuous (Fig. 1c, d) and pulse etching (Fig. 3) processes. The rate of $\mathrm{H}^{+}$reduction over the $\mathrm{Nb}$ surface containing only primary pores, formed through the continuous etching process, was approximately 1.3 times higher than that over the pristine flat $\mathrm{Nb}$ surface. The hierarchical porous structure, produced via the pulse etching, caused a further increase in the rate of $\mathrm{H}^{+}$reduction, which was found to be approximately 3.7 times higher than that over pristine $\mathrm{Nb}$. These findings indicate that the continuous and pulse etching processes reported herein led to a 1.3- and 3.7-fold increase in the $\mathrm{Nb}$ surface area (i.e., roughness factor, $r=1.3$ and 3.7), respectively.

The equation for evaluating the total surface area of a porous material, i.e., Eq. (7), was used to analyze the surface area of the experimentally produced porous $\mathrm{Nb}$ (Fig. 5b). Firstly, the changes in the surface area were calculated as a function of the hierarchical order of the pores for two different, hypothetical porous surfaces. One hypothetical surface consisted of pores with an ideal hemispherical shape with an eccentricity, $e$, of 0 and a $k$ value of 2 . The other was based on the experimentally observed pore shapes, i.e., oblate spheroids with the major axis length (pore diameter) that is twice as great as the minor axis length (pore depth). The corresponding eccentricity, $e$, and $k$ value was chosen to be 0.87 and 1.38 , respectively. The dependence of the surface area on the hierarchical pore order of these two hypothetic cases was presented in dashed lines in Fig. 5b.

Then, an analysis was made on the experimentally produced porous $\mathrm{Nb}$. As a matter of fact, it is virtually impossible to accurately determine the hierarchical pore order of the porous structure obtained via pulse etching due to the ambiguity of the multi-layer pore shape and the limitations of the measuring devices. Nevertheless, based on the surface and side images of the porous structure obtained through an electron microscope, it is observed that at least tertiary pores were formed, although higher-order pore structures may also have been formed (Fig. 3). Thus, while analyzing the surface area of the experimentally obtained porous structure using Eq. (7), we considered the following two cases: (a) the pore order $n$ was assumed to be 3 . Then, the eccentricity $e$ was determined to be approximately 0.73 using the experimental surface area and Eq. (7), i.e., the pores had an oblate spheroid structure, wherein the diameter was approximately 1.45-fold greater than the depth ( $\boldsymbol{A}$ in Fig. 5(b)). (b) It was assumed that the $e$ value of 0.87 , determined for the primary pores, is also applicable to the higher-order pores. The hierarchical order of the porous $\mathrm{Nb}$ structure was accordingly calculated to be 4-5 ( $\boldsymbol{B}$ in Fig. 5(b)). In spite of the above discussion on pore shape and hierarchical pore order of experimentally produced porous $\mathrm{Nb}$ using theoreti- 
cal formula, however, various deviations from the ideal porous structure (e.g. variations of pore shape, size, and aspect ratio) could exist, and the theoretical formulas derived herein include several assumptions. Thus, the proposed theoretical approach should only be used for a general understanding of the pore structures.

\section{Conclusion}

In this work, a hierarchical porous structure was successfully created on $\mathrm{Nb}$ surface by using a onepot, HF-free electrochemical etching process. The following conclusions were made from the experimental and theoretical analyses.

1. Unlike the 1D deep pore created by a conventional electrochemical etching in HF-containing solution, the oblate spheroidal shaped pore whose radius is larger than the depth was formed when the $\mathrm{SO}_{4}{ }^{2-}$ ion was used as an aggressive anion for an electrochemical etching. Such a pore with a relatively open conformation might make it difficult for new smaller pore to be formed on its surface due to the low content of the reactants inside it. This led to the formation of simple porous structure having only primary pore when the etching was conducted using a single continuous current application.

2. It was experimentally proved that pulse etching was quite effective in creating a hierarchical porous or self-similar fractal porous structure with high pore order, most likely due to the repeated supply of the $\mathrm{SO}_{4}{ }^{2-}$ ions to the pore surface during the rest time between etching processes and the resulting high content of reactants favorable for pore nucleation during the subsequent re-etching process. In particular, well-defined hierarchical structure with high pore order (more than 3 ) was produced in conditions of a limited repetition of pulse etching and high concentration of $\mathrm{SO}_{4}{ }^{2-}$.

3. The relation was theoretically obtained for the surface area of porous $\mathrm{Nb}$ and the hierarchical order of pore. It was suggested that the derived formula could give the comprehensive understanding of the actual pore shape and hierarchical pore order especially via the comparative analysis of the theoretical and experimental surface areas. Nevertheless, it needs to be further improved for more precise analysis in terms of reflecting the variations of pore shape, size, aspect ratio, etc.

\section{Acknowledgements}

This work was supported by the National Research Foundation (NFR-2018R1A5A1025594) of the Ministry of Science and ICT.

\section{References}

[1] D. Rosenfeld, P.E. Schmid, S. Szeles, F. Levy, V. Demarne and A. Grisel, Sens. Actuators B Chem., 1996, 37(1-2), 83-89.

[2] L. Chambon, A. Pauly, J.P. Germain, C. Maleysson, V. Demarne and A. Grisel, Sens. Actuators B Chem., 1997, 43(1-3), 60-64.

[3] Z. Wang, Y. Hu, W. Wang, X. Zhang, B. Wang, H. Tian, Y. Wang, J. Guan and H. Gu, Int. J. Hydrogen. Energy, 2012, 37(5), 4526-4532.

[4] R.A. Rani, A.S. Zoolfakar, M.F.M. Ryeeshyam, A.S Ismail, M.H. Mamat, S. Alrokayan, H. Khan, K. Kalantar-Zadeh and M.R. Mahmood, J. Electron. Mater., 2019, 48(6), 3805-3815.

[5] R. Ghosh, M.K. Brennaman, T. Uher, M-R. Ok, E.T. Samulski, L.E. McNeil, T.J. Meyer and R. Lopez, ACS Appl. Mater. Interfaces., 2011, 3(10), 3929-3935.

[6] X. Fang, L. Hu, K. Huo, B. Gao, L. Zhao, M. Liao, P.K. Chu, Y. Bando and D. Golberg, Adv. Funct. Mater., 2011, 21(20), 3907-3915.

[7] K. Kim, J. Park, G. Cha, J.E. Yoo and J. Choi, Mater. Chem. Phys., 2013, 141(2-3), 810-815.

[8] V. Augustyn, P. Simon and B. Dunn, Energy Environ. Sci., 2014, 7(5), 1597-1614.

[9] A.L. Viet, M.V. Reddy, R. Jose, B.V.R. Chowdari and S. Ramakrishna, J. Phys. Chem. C., 2010, 114(1), 664-671.

[10] J.W. Kim, T. Wada, S.G. Kim and H. Kato, Mater. Lett., 2014, 116, 223-226.

[11] J. Come, V. Augustyn, J.W. Kim, P. Rozier, P-L. Taberna, P. Gogotsi, J.W. Long, B. Dunn and P. Simon, J. Electrochem. Soc., 2014, 161(5), 718-725.

[12] R.A. Rani, A.S. Zoolfakar, A.P. O'Mullane, M.W. Austin and K. Kalantar-Zadeh, J. Mater. Chem. A Mater, 2014, 2(38), 15683-15703.

[13] H-C. Shin, J. Dong and M. Liu, Adv. Mater, 2003, 15(19), 1610-1614.

[14] H.-R. Jung, E.-J. Kim, Y.-J. Park and H.-C. Shin, J. Power Sources., 2011, 196(11), 5122-5127.

[15] W.-S. Choi, W. Chang and H.-C. Shin, J. Solid State Electrochem., 2014, 18(2), 427-433.

[16] Y.-M. Chun and H.-C. Shin, Electrochim. Acta, 2016, 209, 369-378.

[17] B. Xu, F. Wu, R. Chen, G. Cao, S. Chen, Z. Zhou and Y. Yang, Electrochem. commun., 2008, 10(5), 795-797.

[18] S. Lou, X. Cheng, L. Wang, J. Gao, Q. Li, Y. Ma, Y. Gao, P. Zuo, C. Du and G. Yin, J. Power Sources., 2017, 361, 80-86.

[19] R.A. Rani, A.S. Zoolfakar, J.Z. Ou, M.R. Field, M. 
Austin and K. Kalantar-zadeh, Sens. Actuators B Chem., 2013, 176, 149-156.

[20] Y.R. Lim, Y. Ko, J. Park, W.I. Cho, S.A. Lim and E.H. Cha, J. Electrochem. Sci. Technol., 2019, 10(1), 89-97.

[21] W. Zhao, W. Choi and W-S. Yoon, J. Electrochem. Sci. Technol., 2019, 11(3), 195-219.

[22] X.W. Lou, L.A. Archer and Z. Yang, Adv. Mater., 2008, 20(21), 3987-4019.

[23] C. Liu, F. Li, L.P. Ma and H.M. Cheng, Adv. Mater, 2010, 22(8), 28-62.

[24] J.Z. Ou, R.A. Rani, M-H Ham, M.R. Field, Y. Zhang, H. Zheng, P. Reece. S. Zhuiykov, S. Sriram, M. Bhaskaran, R.B. Kaner and K. Kalantar-zadeh, ACS nano., 2012, 6(5), 4045-4053.

[25] R.A. Rani, A.S. Zoolfakar, J.Z. Ou, R.A. Kadir, H. Nili, K. Latham, S. Sriram, M. Bhaskaran, S. Zhuiykov, R.B.
Kaner and K. Kalantar-zadeh, ChemComm., 2013, 49(56), 6349-6351.

[26] M. Altomare, G. Cha, P. Schmuki, Electrochim. Acta., 2020, 136158

[27] J. Choi, J.H. Lim, S.C. Lee, J.H. Chang, K.J. Kim and M.A. Cho, Electrochim. Acta., 2006, 51(25), 5502-5507.

[28] K. Lee, Y. Yang, M. Yang and P. Schmuki, Chem. Eur. J., 2012, 18(31), 9521-9524.

[29] J.E. Yoo and J. Choi, Electrochem. commun., 2011, 13(3), 298-301.

[30] Z. Huang, N. Geyer, P. Werner, J. Boor and U. Gösele, Ad.v Mater., 2011, 23(2), 285-308.

[31] J.M. Dura and A. Sarangan, J Micro Nanolithogr MEMS MOEMS., 2017, 16(1), 014502.

[32] S.R. Keller, Math. Comput., 1979, 33(145), 310-314. 